\title{
Oscillation and Nonoscillation of Asymptotically Almost Periodic Half-Linear Difference Equations
}

\author{
Michal Veselý ${ }^{1}$ and Petr Hasil ${ }^{2}$ \\ ${ }^{1}$ Department of Mathematics and Statistics, Masaryk University, Kotlárská 2, 61137 Brno, Czech Republic \\ ${ }^{2}$ Department of Mathematics, Mendel University in Brno, Zemědělská 1, 61300 Brno, Czech Republic \\ Correspondence should be addressed to Michal Veselý; michal.vesely@mail.muni.cz
}

Received 18 March 2013; Accepted 20 May 2013

Academic Editor: Ondrej Dosly

Copyright (C) 2013 M. Veselý and P. Hasil. This is an open access article distributed under the Creative Commons Attribution License, which permits unrestricted use, distribution, and reproduction in any medium, provided the original work is properly cited.

We analyse half-linear difference equations with asymptotically almost periodic coefficients. Using the adapted Riccati transformation, we prove that these equations are conditionally oscillatory. We explicitly find a constant, determined by the coefficients of a given equation, which is the borderline between the oscillation and the nonoscillation of the equation. We also mention corollaries of our result with several examples.

\section{Introduction}

We intend to study the oscillation behaviour of the secondorder half-linear difference equation

$$
\Delta\left[r_{k} \Phi\left(\Delta y_{k}\right)\right]+p_{k} \Phi\left(y_{k+1}\right)=0,
$$

where $\Phi(y)=|y|^{p-1} \operatorname{sgn} y, p>1$, and $r_{k} \neq 0$ for all considered $k$. Throughout this paper, we consider integers $k \geq m$ for a sufficiently large $m \in \mathbb{N}$. Concerning the oscillation and nonoscillation of these equations, many results have been obtained in the last years. The basic facts of the oscillation theory for (1) are established in [1]. Several criteria for (non)oscillation of (1) are proved, for example, in [2-6] (for (1) with $r_{k} \equiv 1$, see [7-9]; for the continuous case, see [1012]). Oscillatory properties of slightly more general difference equations are investigated, for example, in $[13,14]$. A detailed literature overview can be found in [15].

The oscillation theory of half-linear difference equations is motivated by the continuous case and by the linear case.

Equation (1) is the discretization of the half-linear differential equation

$$
\left[r(t) \Phi\left(y^{\prime}(t)\right)\right]^{\prime}+p(t) \Phi(y(t))=0, \quad r(t)>0 .
$$

The first occurrence of an equation of this form is dated back to 1961 (see [16]). But $[17,18]$ are regarded as the basic papers in this field. For a comprehensive overview of halflinear differential equations, we refer to [19] with references cited therein. Note that techniques needed in the discrete theory are often more complicated than their continuous counterparts. In this paper, we apply the Riccati method which is used in the continuous case as well. Comparing this paper with its continuous counterpart [20], one can see that the discrete method requires several nontrivial extra steps and is technically more exacting. Particularly, we cannot use the fact that the ranges of considered solutions are intervals.

Equation (1) is a generalization of the linear equation

$$
\Delta\left(r_{k} \Delta y_{k}\right)+p_{k} y_{k+1}=0, \quad r_{k} \neq 0,
$$

whose oscillation theory is well described in the literature (see $[21,22]$ and references therein). There are limitations of the tools known from the theory of linear equations (e.g., the transformation theory and the Casoratian identity). These limitations are more or less consequences of the fact that the solution space of half-linear equations is not additive. Nevertheless, it remains homogeneous (likewise in the linear case). This observation motivates the term "half-linear."

Actually, we consider (1) in the form

$$
\Delta\left[r_{k} \Phi\left(\Delta y_{k}\right)\right]+\frac{\gamma s_{k}}{(k+1)^{(p)}} \Phi\left(y_{k+1}\right)=0,
$$


where $\left\{r_{k}\right\},\left\{s_{k}\right\}$ are positive sequences, $\gamma>0$, and $k^{(p)}$ stands for the so-called generalized power function. Our objective is to prove that (4) is conditionally oscillatory and to find the corresponding oscillation constant. We recall that (4) is conditionally oscillatory if there exists $\Gamma>0$ with the property that (4) is oscillatory for $\gamma>\Gamma$ and nonoscillatory for $\gamma<\Gamma$. In this case, $\Gamma$ is called the oscillation constant of (4). Note that we consider the form with the generalized power function $1 /(k+1)^{(p)}$ because we want to be consistent with previous results and our method is much more transparent in this case.

According to the best of our knowledge, the first result about the conditional oscillation of a second-order equation was proved by Kneser back in 1893 (see [23]). In [23], it is shown that the differential equation

$$
y^{\prime \prime}(t)+\frac{\gamma}{t^{2}} y(t)=0
$$

is conditionally oscillatory with the oscillation constant $\Gamma=$ $1 / 4$. The corresponding difference equation

$$
\Delta^{2} y_{k}+\frac{\gamma}{(k+1) k} y_{k+1}=0
$$

is conditionally oscillatory with the oscillation constant $\Gamma=$ $1 / 4$ as well (see [24]). In [25], the previous Kneser result is generalized for the equation

$$
\left[r(t) y^{\prime}(t)\right]^{\prime}+\frac{\gamma s(t)}{t^{2}} y(t)=0
$$

where $r, s: \mathbb{R} \rightarrow \mathbb{R}$ are positive periodic continuous functions. We can also mention [26] and more generally [27, 28]. For the discrete linear equation

$$
\Delta\left(r_{k} \Delta y_{k}\right)+\frac{\gamma s_{k}}{(k+1) k} y_{k+1}=0,
$$

we refer to [29], where the corresponding result is obtained. Nevertheless, the first discrete version of the result of [25] was proved in [30]. The oscillation constant from [30] coincides with the one from [29] if the considered coefficients are asymptotically constant. In fact, the conditional oscillation of (8) with almost periodic coefficients is proved in [29]. The oscillation constant for the periodic half-linear equation

$$
\left[r(t) \Phi\left(y^{\prime}(t)\right)\right]^{\prime}+\frac{\gamma s(t)}{t^{p}} \Phi(y(t))=0
$$

is derived in [31] (see also [32]) and the oscillation constant for (9) with asymptotically almost periodic coefficients is obtained in [20]. Our aim is to prove the discrete counterpart of the main result of [20]; that is, we find the oscillation constant for (4) with asymptotically almost periodic coefficients. We add that this result is new in the periodic case and in the linear case as well.

The paper is organized as follows. In Section 2, we recall elements of the theory of almost periodic and asymptotically almost periodic sequences, which we will use later. In Section 3, we mention basic definitions from the oscillation theory and we derive the adapted Riccati equation. Using the prepared Riccati technique, we prove the announced result in Section 4 . Then, we state its corollaries and we illustrate them by examples. We also show a way how the main result can be applied for equations with coefficients which change their signs.

Henceforth, let $p>1$ be arbitrarily given and let $q \in \mathbb{R}$ be such that

$$
\frac{1}{p}+\frac{1}{q}=1, \quad \text { i.e., } p+q=p q \text {. }
$$

Thus, for $\Phi(x)=|x|^{p-1} \operatorname{sgn} x$, we have $\Phi^{-1}(x)=|x|^{q-1} \operatorname{sgn} x$. For the reader's convenience, we recall the generalized power function (sometimes called the falling factorial power)

$$
k^{(\alpha)}=\frac{\Gamma(k+1)}{\Gamma(k+1-\alpha)}, \quad \alpha \in(-1, k), k \in \mathbb{N},
$$

where

$$
\Gamma(x)=\int_{0}^{\infty} e^{-s} s^{x-1} d s, \quad x>1,
$$

and the identities

$$
\begin{array}{r}
\Delta k^{(\alpha+1)}=(\alpha+1) k^{(\alpha)}, \quad \frac{k^{(\alpha+1)}}{k^{(\alpha)}}=k-\alpha, \\
\alpha \in(-1, k), \quad k \in \mathbb{N},
\end{array}
$$

which we will often apply later. We also refer to [22, Chapter 2].

\section{Asymptotic Almost Periodicity}

We begin with the notion of almost periodicity. The fundamental results of the theory of almost periodic sequences can be found, for example, in [33, Section I.6].

Definition 1. A sequence $\left\{f_{k}\right\}_{k \in \mathbb{Z}} \subset \mathbb{R}$ is called almost periodic if, for every $\varepsilon>0$, there exists an integer $p(\varepsilon)$ with the property that any set consisting of $p(\varepsilon)$ consecutive integers contains at least one number $l$ for which

$$
\left|f_{k+l}-f_{k}\right|<\varepsilon, \quad k \in \mathbb{Z}
$$

Definition 2. A continuous function $f: \mathbb{R} \rightarrow \mathbb{R}$ is called almost periodic if, for every $\varepsilon>0$, there exists a number $p(\varepsilon)>$ 0 such that any interval of length $p(\varepsilon)$ of the real line contains at least one point $s$ satisfying

$$
|f(t+s)-f(t)|<\varepsilon, \quad t \in \mathbb{R} .
$$

Theorem 3. A necessary and sufficient condition for a sequence $\left\{f_{k}\right\}_{k \in \mathbb{Z}} \subset \mathbb{R}$ to be almost periodic is the existence of an almost periodic function $g: \mathbb{R} \rightarrow \mathbb{R}$ with the property that $f_{k}=g(k)$ for all $k \in \mathbb{Z}$.

Proof. See [33, Theorem 1.27]. 
Theorem 4. Any almost periodic sequence is bounded.

Proof. The statement of the theorem follows from Theorem 3 and from the boundedness of any almost periodic function on the real line (see, e.g., [33, Theorem 1.2]).

Theorem 5. A sequence $\left\{f_{k}\right\}_{k \in \mathbb{Z}} \subset \mathbb{R}$ is almost periodic if and only if any sequence of the form $\left\{\left\{f_{k+h(n)}\right\}_{k \in \mathbb{Z}}\right\}_{n \in \mathbb{N}}$, where $h(n) \in$ $\mathbb{Z}$, has a uniformly convergent subsequence with respect to $k$.

Proof. See [33, Theorem 1.26].

The following result is a consequence of Theorems 4 and 5 .

Corollary 6. The sum and the product of two almost periodic sequences are almost periodic as well.

Theorem 7. If $\left\{f_{k}\right\}_{k \in \mathbb{Z}}$ is almost periodic, then the limit

$$
\lim _{k \rightarrow \infty} \frac{1}{k} \sum_{i=j}^{j+k-1} f_{i}
$$

is finite and exists uniformly with respect to $j \in \mathbb{Z}$.

Proof. See [33, Theorem 1.28].

Theorem 8. If $\left\{f_{k}\right\}_{k \in \mathbb{Z}} \subset \mathbb{R}$ is almost periodic and $F: \mathbb{R} \rightarrow \mathbb{R}$ is a uniformly continuous function on the set $\left\{f_{k} ; k \in \mathbb{Z}\right\}$, then the composition $\left\{F\left(f_{k}\right)\right\}_{k \in \mathbb{Z}}$ is almost periodic.

Proof. The theorem follows, for example, from [34, Theorem 2.4].

As a direct generalization of almost periodicity, we consider the asymptotic almost periodicity. We refer to [35], where asymptotically almost periodic sequences were introduced with their basic characteristics.

Definition 9. We say that a sequence $\left\{f_{k}\right\}_{k \in \mathbb{N}} \subset \mathbb{R}$ is asymptotically almost periodic if it can be represented in the form $f_{k}=$ $f_{k}^{1}+f_{k}^{2}, k \in \mathbb{N}$, where $\left\{f_{k}^{1}\right\}_{k \in \mathbb{Z}}$ is almost periodic and $\left\{f_{k}^{2}\right\}_{k \in \mathbb{N}}$ has the property that $\lim _{k \rightarrow \infty} f_{k}^{2}=0$. The sequence $\left\{f_{k}^{1}\right\}$ is called the almost periodic part of $\left\{f_{k}\right\}$.

Considering Theorem 7, we immediately obtain the following.

Corollary 10. Let an asymptotically almost periodic sequence $\left\{f_{k}\right\}_{k \in \mathbb{N}}$ be given, and let $\left\{f_{k}^{1}\right\}_{k \in \mathbb{Z}}$ be its almost periodic part. The limit

$$
M\left(\left\{f_{k}\right\}\right):=\lim _{k \rightarrow \infty} \frac{1}{k} \sum_{i=j}^{j+k-1} f_{i}=\lim _{k \rightarrow \infty} \frac{1}{k} \sum_{i=j}^{j+k-1} f_{i}^{1}
$$

is finite and exists uniformly with respect to $j \in \mathbb{N}$.

Definition 11. The number $M\left(\left\{f_{k}\right\}\right)$ introduced in (17) is called the mean value of an asymptotically almost periodic sequence $\left\{f_{k}\right\}_{k \in \mathbb{N}}$.
Remark 12. According to Definition 1 and (17), we have $M\left(\left\{f_{k}\right\}\right)>0$ for any positive asymptotically almost periodic sequence $\left\{f_{k}\right\}_{k \in \mathbb{N}}$ with the property that $\limsup _{k \rightarrow \infty} f_{k}>0$.

Corollary 13. If $\left\{f_{k}\right\}_{k \in \mathbb{N}}$ is an asymptotically almost periodic sequence satisfying

$$
\inf \left\{f_{k} ; k \in \mathbb{N}\right\}>0 \text {, }
$$

then the sequence $\left\{g_{k}\right\}_{k \in \mathbb{N}}$, defined by the formula $g_{k}=f_{k}^{\alpha}$, is asymptotically almost periodic for arbitrarily given $\alpha \in \mathbb{R}$.

Proof. Let $f_{k}=f_{k}^{1}+f_{k}^{2}, k \in \mathbb{N}$, where $\left\{f_{k}^{1}\right\}_{k \in \mathbb{Z}}$ is the almost periodic part of $\left\{f_{k}\right\}$. Considering Definition 1, we have

$$
\inf \left\{f_{k}^{1} ; k \in \mathbb{Z}\right\}=\inf \left\{f_{k}^{1} ; k \in \mathbb{Z}, k \geq a\right\}, \quad a \in \mathbb{R},
$$

and therefore (see (18))

$$
\inf \left\{f_{k}^{1} ; k \in \mathbb{Z}\right\} \geq \inf \left\{f_{k} ; k \in \mathbb{N}\right\}>0 .
$$

If we put $g_{k}^{1}:=\left[f_{k}^{1}\right]^{\alpha}, k \in \mathbb{Z}$, then Theorems 4 and 8 imply the almost periodicity of $\left\{g_{k}^{1}\right\}_{k \in \mathbb{Z}}$. Since

$$
\lim _{k \rightarrow \infty}\left(g_{k}-g_{k}^{1}\right)=\lim _{k \rightarrow \infty}\left(\left[f_{k}^{1}+f_{k}^{2}\right]^{\alpha}-\left[f_{k}^{1}\right]^{\alpha}\right)=0,
$$

we know that $\left\{g_{k}\right\}$ can be expressed as the sum of the almost periodic sequence $\left\{g_{k}^{1}\right\}$ and the sequence $\left\{g_{k}-g_{k}^{1}\right\}$ vanishing at infinity. It means that $\left\{g_{k}\right\}$ is asymptotically almost periodic.

\section{Half-Linear Riccati Equation}

In this section, we recall the oscillation behaviour of halflinear difference equations and we introduce the (adapted) half-linear Riccati equation. For more details, we refer to [15, Chapter 3] or [19, Chapter 8] (with references cited therein). We remark that the (adapted) Riccati technique is an essential tool to prove the following given Theorem 17.

Let us consider the equation

$$
\Delta\left[r_{k} \Phi\left(\Delta x_{k}\right)\right]+p_{k} \Phi\left(x_{k+1}\right)=0,
$$

where $r_{k}, p_{k}>0$ for all considered $k$ and the sequence $\left\{r_{k}\right\}$ is bounded. We say that an interval $(l, l+1], l \in \mathbb{N}$, contains the generalized zero of a solution $\left\{x_{k}\right\}$ of (22) if $x_{l} \neq 0$ and $x_{l} x_{l+1} \leq 0$. Equation (22) is said to be disconjugate on a set $\{l, l+1, \ldots, l+n\}$ provided any solution of $(22)$ has at most one generalized zero on $(l, l+n+1]$ and the solution $\left\{\tilde{x}_{k}\right\}$ given by the initial value $\tilde{x}_{l}=0$ has no generalized zero on $(l, l+n+1]$. Otherwise, $(22)$ is said to be conjugate on $\{l, l+1, \ldots, l+n\}$.

The Sturm type separation theorem is valid for (22) (see [1, Theorem 3]). Thus, the oscillation of an arbitrary solution of (22) implies the oscillation of all solutions of (22). It means that we can categorize (22) as oscillatory or nonoscillatory.

Definition 14. Equation (22) is called nonoscillatory if there exists $n \in \mathbb{N}$ with the property that (22) is disconjugate on $\{n, n+1, \ldots, n+i\}$ for all $i \in \mathbb{N}$. In the opposite case, $(22)$ is called oscillatory. 
Now, we turn our attention to the half-linear Riccati equation. Using the substitution

$$
w_{k}=r_{k} \Phi\left(\frac{\Delta x_{k}}{x_{k}}\right)
$$

we transform (22) into

$$
\Delta w_{k}+p_{k}+w_{k}\left(1-\frac{r_{k}}{\Phi\left[\Phi^{-1}\left(r_{k}\right)+\Phi^{-1}\left(w_{k}\right)\right]}\right)=0
$$

According to $\left[15\right.$, Lemma 3.2.6, $\left.\left(\mathrm{I}_{8}\right)\right]$, if $w_{k}+r_{k}>0$, then

$$
\begin{gathered}
w_{k}\left(1-\frac{r_{k}}{\Phi\left[\Phi^{-1}\left(r_{k}\right)+\Phi^{-1}\left(w_{k}\right)\right]}\right) \\
=\frac{(p-1)\left|w_{k}\right|^{q}\left|\beta_{k}\right|^{p-2}}{\Phi\left[\Phi^{-1}\left(r_{k}\right)+\Phi^{-1}\left(w_{k}\right)\right]}
\end{gathered}
$$

where $\beta_{k}$ is between $\Phi^{-1}\left(r_{k}\right)$ and $\Phi^{-1}\left(r_{k}\right)+\Phi^{-1}\left(w_{k}\right)$; that is, for $w_{k}+r_{k}>0$, we can rewrite (24) in the following form:

$$
\Delta w_{k}+p_{k}+\frac{(p-1)\left|w_{k}\right|^{q}\left|\beta_{k}\right|^{p-2}}{\Phi\left[\Phi^{-1}\left(r_{k}\right)+\Phi^{-1}\left(w_{k}\right)\right]}=0
$$

Next, putting

$$
\zeta_{k}=-k^{(p-1)} w_{k}
$$

we obtain

$$
\Delta \zeta_{k}=-\Delta\left(k^{(p-1)} w_{k}\right)=-(p-1) k^{(p-2)} w_{k}-(k+1)^{(p-1)} \Delta w_{k},
$$

and then, applying (26), we obtain the adapted Riccati equation

$$
\begin{aligned}
\Delta \zeta_{k}= & \frac{1}{k-p+2} \\
& \times\left[(p-1) \zeta_{k}+(k+1)^{(p)} p_{k}\right. \\
& \left.\quad+\frac{(k+1)(p-1)\left|\beta_{k}\right|^{p-2}\left|\zeta_{k}\right|^{q}}{\left[k^{(p-1)}\right]^{q-1} \Phi\left[\Phi^{-1}\left(r_{k}\right)+\Phi^{-1}\left(-\zeta_{k} / k^{(p-1)}\right)\right]}\right],
\end{aligned}
$$

where $\beta_{k}$ is between $\Phi^{-1}\left(r_{k}\right)$ and $\Phi^{-1}\left(r_{k}\right)+\Phi^{-1}\left(-\zeta_{k} / k^{(p-1)}\right)$.

\section{Conditional Oscillation}

To prove the announced theorem, we need the following two lemmas.

Lemma 15. If (22) is nonoscillatory, then there exists a negative solution $\left\{\zeta_{k}\right\}_{k \geq k_{0}}$ of (29) such that

$$
\lim _{k \rightarrow \infty} \frac{\zeta_{k}}{k^{(p-1)}}=0
$$

Proof. Applying [1, Theorem 1] (see also [15, Theorem 3.3.4]), we know that the nonoscillation of (22) implies the existence of a solution $\left\{w_{k}\right\}_{k \geq k_{0}}$ of (26) satisfying $w_{k}+r_{k}>0, k \geq k_{0}$. Considering [36, Lemma 1, (v) and Theorem 1], we obtain $\lim _{k \rightarrow \infty} w_{k}=0$. Since the sequence $\left\{w_{k}\right\}$ is decreasing (see directly (26)), we have $w_{k}>0$ for all $k \geq k_{0}$. Thus, there exists a negative solution $\left\{\zeta_{k}\right\}_{k \geq k_{0}}$ of (29). Finally, (27) gives

$$
\lim _{k \rightarrow \infty} \frac{\zeta_{k}}{k^{(p-1)}}=-\lim _{k \rightarrow \infty} w_{k}=0
$$

Lemma 16. If there exists a negative solution $\left\{\zeta_{k}\right\}_{k \geq k_{0}}$ of (29), then (22) is nonoscillatory.

Proof. The statement of the lemma follows from [1, Theorem 1].

Now we can formulate and prove the main result.

Theorem 17. Let $\gamma \in \mathbb{R}^{+}$be given, and let $\left\{r_{k}\right\}_{k \in \mathbb{N}}$ and $\left\{s_{k}\right\}_{k \in \mathbb{N}}$ be arbitrary positive asymptotically almost periodic sequences such that

$$
\inf \left\{r_{k} ; k \in \mathbb{N}\right\}>0, \quad \limsup _{k \rightarrow \infty} s_{k}>0 \text {. }
$$

Let

$$
\Gamma:=q^{-p}\left[M\left(\left\{r_{k}^{1-q}\right\}\right)\right]^{1-p}\left[M\left(\left\{s_{k}\right\}\right)\right]^{-1} .
$$

Consider the equation

$$
\Delta\left[r_{k} \Phi\left(\Delta x_{k}\right)\right]+\frac{\gamma s_{k}}{(k+1)^{(p)}} \Phi\left(x_{k+1}\right)=0 .
$$

If $\gamma>\Gamma$, then (34) is oscillatory. If $\gamma<\Gamma$, then (34) is nonoscillatory.

Proof. We put (see Theorem 4)

$$
\begin{aligned}
r^{-} & :=\inf \left\{r_{k} ; k \in \mathbb{N}\right\}, \\
r^{+} & :=\sup \left\{r_{k} ; k \in \mathbb{N}\right\}, \\
s^{+} & :=\sup \left\{s_{k} ; k \in \mathbb{N}\right\} .
\end{aligned}
$$

For an arbitrarily given number $\gamma \neq \Gamma$, we will consider an integer $\alpha \geq 2$ such that

$$
\begin{gathered}
\frac{1}{\alpha} \sum_{i=k}^{k+\alpha-1} s_{i}>\frac{M\left(\left\{s_{k}\right\}\right)}{2}>0, \quad k \in \mathbb{N}, \\
\left|\Gamma-q^{-p}\left[\frac{1}{\alpha} \sum_{i=k}^{k+\alpha-1} r_{i}^{1-q}\right]^{1-p}\left[\frac{1}{\alpha} \sum_{i=k}^{k+\alpha-1} s_{i}\right]^{-1}\right|<\frac{|\Gamma-\gamma|}{2}, \quad k \in \mathbb{N} .
\end{gathered}
$$

That is,

$$
\left|\gamma-q^{-p}\left[\frac{1}{\alpha} \sum_{i=k}^{k+\alpha-1} r_{i}^{1-q}\right]^{1-p}\left[\frac{1}{\alpha} \sum_{i=k}^{k+\alpha-1} s_{i}\right]^{-1}\right|>\frac{|\gamma-\Gamma|}{2}, \quad k \in \mathbb{N} .
$$


From Remark 12 and Corollaries 10 and 13, we know that such a number $\alpha$ exists.

At first, we consider the oscillatory part of the theorem. By contradiction, we suppose that $\gamma>\Gamma$ is given and that (34) is nonoscillatory. Lemma 15 says that there exists a negative solution $\left\{\zeta_{k}\right\}_{k \geq k_{0}}$ of the equation

$$
\begin{aligned}
\Delta \zeta_{k}= & \frac{1}{k-p+2} \\
& \times\left[(p-1) \zeta_{k}+\gamma s_{k}\right. \\
& \left.\quad+\frac{(k+1)(p-1)\left|\beta_{k}\right|^{p-2}\left|\zeta_{k}\right|^{q}}{\left[k^{(p-1)}\right]^{q-1} \Phi\left[\Phi^{-1}\left(r_{k}\right)+\Phi^{-1}\left(-\zeta_{k} / k^{(p-1)}\right)\right]}\right]
\end{aligned}
$$

where

$$
\Phi^{-1}\left(r_{k}\right) \leq \beta_{k} \leq \Phi^{-1}\left(r_{k}\right)+\Phi^{-1}\left(-\frac{\zeta_{k}}{k^{(p-1)}}\right), \quad k \geq k_{0} .
$$

Note that (39) is the adapted Riccati equation associated with (34) and that (30) holds. Particularly, from (30), we know

$$
\lim _{k \rightarrow \infty} \frac{\Phi\left[\Phi^{-1}\left(r_{k}\right)+\Phi^{-1}\left(-\zeta_{k} / k^{(p-1)}\right)\right]}{r_{k}}=1
$$

and (see (40))

$$
\lim _{k \rightarrow \infty} \frac{\beta_{k}}{\Phi^{-1}\left(r_{k}\right)}=\lim _{k \rightarrow \infty} \frac{\beta_{k}}{r_{k}^{q-1}}=1 .
$$

Using (consider, e.g., the Stirling formula)

$$
\lim _{k \rightarrow \infty} \frac{k^{a}}{k^{(a)}}=\lim _{k \rightarrow \infty} \frac{k^{a} \Gamma(k+1-a)}{\Gamma(k+1)}=1, \quad a>0,
$$

we also obtain

$$
\lim _{k \rightarrow \infty} \frac{k+1}{\left[k^{(p-1)}\right]^{q-1}}=\lim _{k \rightarrow \infty} \frac{k+1}{\left[k^{p-1}\right]^{q-1}}=\lim _{k \rightarrow \infty} \frac{k+1}{k}=1 .
$$

Combining (39), (41), (42), and (44), we can find an integer $\widetilde{k} \geq k_{0}$ with the property that

$$
\begin{aligned}
\Delta \zeta_{k} & \geq \frac{1}{k-p+2}\left[(p-1) \zeta_{k}+\frac{1}{2} \frac{(p-1) r_{k}^{[q-1][p-2]}\left|\zeta_{k}\right|^{q}}{r_{k}}\right] \\
& =\frac{p-1}{k-p+2}\left[\zeta_{k}+\frac{1}{2} r_{k}^{1-q}\left|\zeta_{k}\right|^{q}\right] \\
& \geq \frac{p-1}{k-p+2}\left[\zeta_{k}+\frac{\left|\zeta_{k}\right|^{q}}{2\left[r^{+}\right]^{q-1}}\right]
\end{aligned}
$$

and that

$$
\begin{aligned}
\Delta \zeta_{k} \leq & \frac{1}{k-p+2}\left[\gamma s^{+}+2 \frac{(p-1) r_{k}^{[q-1][p-2]}\left|\zeta_{k}\right|^{q}}{r_{k}}\right] \\
= & \frac{1}{k-p+2}\left[\gamma s^{+}+\frac{2(p-1)\left|\zeta_{k}\right|^{q}}{r_{k}^{q-1}}\right] \leq \frac{1}{k-p+2} \\
& \times\left[\gamma s^{+}+\frac{2(p-1)\left|\zeta_{k}\right|^{q}}{\left[r^{-}\right]^{q-1}}\right]
\end{aligned}
$$

whenever $k \geq \tilde{k}$. Let $\zeta_{k}<-2^{1 /(q-1)} r^{+}$for some $k \geq \tilde{k}$. In this case, we estimate

$$
\left|\zeta_{k}\right|^{q}=\left|\zeta_{k}\right| \cdot\left|\zeta_{k}\right|^{q-1}>\left|\zeta_{k}\right| \cdot\left|2^{1 /(q-1)} r^{+}\right|^{q-1}=\left|\zeta_{k}\right| \cdot 2\left[r^{+}\right]^{q-1}
$$

and hence, by (45), we get $\Delta \zeta_{k}>0$. Thus, there exists $L>0$ satisfying

$$
\zeta_{k} \in(-L, 0), \quad k \geq k_{0} .
$$

Therefore (see (45) and (46)), we have

$$
\left|\Delta \zeta_{k}\right|<\frac{K}{k-p+2}, \quad k \geq \tilde{k}
$$

where

$$
K:=(p-1) L+\gamma s^{+}+\frac{2(p-1) L^{q}}{\left[r^{-}\right]^{q-1}} .
$$

Particularly, (49) implies

$$
\left|\zeta_{k+i}-\zeta_{k+j}\right|<\frac{(\alpha-1) K}{k-p+2}, \quad i, j \in\{0, \ldots, \alpha-1\}, k \geq \widetilde{k}
$$

We define

$$
\begin{gathered}
\xi_{k}:=\frac{1}{\alpha} \sum_{i=k}^{k+\alpha-1} \zeta_{i}, \quad k \geq k_{0}, \\
X_{k}:=(p-1)\left(\frac{p^{k+\alpha-1}}{\alpha} \sum_{i=k}^{1-q} r_{i}^{1-q},\right. \\
Y_{k}:=\left|\xi_{k}\right|\left(\frac{p^{k+\alpha}}{\alpha} \sum_{i=k}^{k+1} r_{i}^{1-q}\right)^{1 / q}, \quad k \geq k_{0} .
\end{gathered}
$$

From (48), we can see

$$
\xi_{k} \in(-L, 0), \quad k \geq k_{0} .
$$


For $k \geq k_{0}$, we compute (see (39))

$$
\begin{aligned}
& \Delta \xi_{k}=\frac{1}{\alpha} \sum_{i=k}^{k+\alpha-1} \Delta \zeta_{i} \\
& =\frac{1}{\alpha} \sum_{i=k}^{k+\alpha-1} \frac{1}{i-p+2} \\
& \times\left[(p-1) \zeta_{i}+\gamma s_{i}\right. \\
& \left.+\frac{(i+1)(p-1)\left|\beta_{i}\right|^{p-2}\left|\zeta_{i}\right|^{q}}{\left[i^{(p-1)}\right]^{q-1} \Phi\left[\Phi^{-1}\left(r_{i}\right)+\Phi^{-1}\left(-\zeta_{i} / i^{(p-1)}\right)\right]}\right] \\
& \geq \frac{1}{k+\alpha-p+1} \\
& \times\left[(p-1) \xi_{k}+\frac{\gamma^{k+\alpha-1}}{\alpha} \sum_{i=k} s_{i}\right. \\
& \left.+\frac{1}{\alpha} \sum_{i=k}^{k+\alpha-1} \frac{(i+1)(p-1)\left|\beta_{i}\right|^{p-2}\left|\zeta_{i}\right|^{q}}{[i(p-1)]^{q-1} \Phi\left[\Phi^{-1}\left(r_{i}\right)+\Phi^{-1}\left(-\zeta_{i} / i^{(p-1)}\right)\right]}\right] \\
& =\frac{1}{k+\alpha-p+1} \\
& \times\left[(p-1) \xi_{k}+\frac{X_{k}^{p}}{p}+\frac{Y_{k}^{q}}{q}+\frac{\gamma}{\alpha} \sum_{i=k}^{k+\alpha-1} s_{i}-\frac{X_{k}^{p}}{p}\right. \\
& +\frac{1}{\alpha} \sum_{i=k}^{k+\alpha-1} \frac{(i+1)(p-1)\left|\beta_{i}\right|^{p-2}\left|\zeta_{i}\right|^{q}}{\left[i^{(p-1)}\right]^{q-1} \Phi\left[\Phi^{-1}\left(r_{i}\right)+\Phi^{-1}\left(-\zeta_{i} / i^{(p-1)}\right)\right]} \\
& \left.-\frac{Y_{k}^{q}}{q}\right]
\end{aligned}
$$

if $\Delta \xi_{k}>0$, or

$$
\begin{aligned}
\Delta \xi_{k} \geq & \frac{1}{k-p+2} \\
& \times\left[(p-1) \xi_{k}+\frac{X_{k}^{p}}{p}+\frac{Y_{k}^{q}}{q}+\frac{\gamma^{k+\alpha-1}}{\alpha} \sum_{i=k} s_{i}-\frac{X_{k}^{p}}{p}\right. \\
& +\frac{1}{\alpha} \sum_{i=k}^{k+\alpha-1} \frac{(i+1)(p-1)\left|\beta_{i}\right|^{p-2}\left|\zeta_{i}\right|^{q}}{[i(p-1)]^{q-1} \Phi\left[\Phi^{-1}\left(r_{i}\right)+\Phi^{-1}\left(-\zeta_{i} / i(p-1)\right)\right]} \\
& \left.\quad-\frac{Y_{k}^{q}}{q}\right]
\end{aligned}
$$

if $\Delta \xi_{k}<0$.
Since

$$
X_{k} Y_{k}=\left|\xi_{k}\right|(p-1)=-\xi_{k}(p-1), \quad k \geq k_{0}
$$

it holds

$$
(p-1) \xi_{k}+\frac{X_{k}^{p}}{p}+\frac{Y_{k}^{q}}{q}=\frac{X_{k}^{p}}{p}+\frac{Y_{k}^{q}}{q}-X_{k} Y_{k} \geq 0, \quad k \geq k_{0}
$$

which follows from the Young inequality.

Using (36), (37), and (38), we have

$$
\begin{aligned}
& \frac{\gamma^{k+\alpha-1}}{\alpha} \sum_{i=k} s_{i}-\frac{X_{k}^{p}}{p}=\frac{\gamma^{k+\alpha-1}}{\alpha} \sum_{i=k} s_{i}-\frac{(p-1)^{p}}{p}\left(\frac{p^{k+\alpha-1}}{\alpha} \sum_{i=k}^{1-q} r_{i}^{-p / q}\right. \\
& =\frac{1}{\alpha} \sum_{i=k}^{k+\alpha-1} s_{i}\left[\gamma-\left(\frac{p-1}{p}\right)^{p}\right. \\
& \times\left(\frac{1}{\alpha} \sum_{i=k}^{k+\alpha-1} r_{i}^{1-q}\right)^{-p / q} \\
& \left.\times\left(\frac{1}{\alpha} \sum_{i=k}^{k+\alpha-1} s_{i}\right)^{-1}\right] \\
& =\frac{1}{\alpha} \sum_{i=k}^{k+\alpha-1} s_{i}\left[\gamma-q^{-p}\left(\frac{1}{\alpha} \sum_{i=k}^{k+\alpha-1} r_{i}^{1-q}\right)^{1-p}\right. \\
& \left.\times\left(\frac{1}{\alpha} \sum_{i=k}^{k+\alpha-1} s_{i}\right)^{-1}\right] \\
& >\frac{M\left(\left\{s_{k}\right\}\right)}{2} \frac{\gamma-\Gamma}{2}, \quad k \geq k_{0} .
\end{aligned}
$$

That is,

$$
\frac{\gamma^{k+\alpha-1}}{\alpha} \sum_{i=k} s_{i}-\frac{X_{k}^{p}}{p}>\frac{M\left(\left\{s_{k}\right\}\right)[\gamma-\Gamma]}{4}>0, \quad k \geq k_{0} .
$$

It remains to estimate the following:

$$
\begin{gathered}
\frac{1}{\alpha} \sum_{i=k}^{k+\alpha-1} \frac{(i+1)(p-1)\left|\beta_{i}\right|^{p-2}\left|\zeta_{i}\right|^{q}}{\left[i^{(p-1)}\right]^{q-1} \Phi\left[\Phi^{-1}\left(r_{i}\right)+\Phi^{-1}\left(-\zeta_{i} / i^{(p-1)}\right)\right]}-\frac{Y_{k}^{q}}{q} \\
\quad=\frac{1}{\alpha} \sum_{i=k}^{k+\alpha-1} \frac{(i+1)(p-1)\left|\beta_{i}\right|^{p-2}\left|\zeta_{i}\right|^{q}}{[i(p-1)]^{q-1} \Phi\left[\Phi^{-1}\left(r_{i}\right)+\Phi^{-1}\left(-\zeta_{i} / i^{(p-1)}\right)\right]}
\end{gathered}
$$




$$
\begin{aligned}
& \quad-\frac{\left|\xi_{k}\right|^{q}}{q} \frac{p^{k+\alpha-1}}{\alpha} \sum_{i=k}^{1-q} r_{i}^{1-q} \\
& =\frac{p-1}{\alpha} \\
& \quad \times \sum_{i=k}^{k+\alpha-1}\left(\begin{array}{c}
\left|\zeta_{i}\right|^{q} \frac{(i+1)\left|\beta_{i}\right|^{p-2}}{[i(p-1)]^{q-1} \Phi\left[\Phi^{-1}\left(r_{i}\right)+\Phi^{-1}\left(-\zeta_{i} / i^{(p-1)}\right)\right]} \\
-\left|\xi_{k}\right|^{q} r_{i}^{1-q}
\end{array}\right), \quad k \geq k_{0} .
\end{aligned}
$$

Applying (41), (42), and (44), we can express

$$
\begin{aligned}
& \frac{1}{\alpha} \sum_{i=k}^{k+\alpha-1} \frac{(i+1)(p-1)\left|\beta_{i}\right|^{p-2}\left|\zeta_{i}\right|^{q}}{[i(p-1)]^{q-1} \Phi\left[\Phi^{-1}\left(r_{i}\right)+\Phi^{-1}\left(-\zeta_{i} / i^{(p-1)}\right)\right]}-\frac{Y_{k}^{q}}{q} \\
& =\frac{p-1}{\alpha} \sum_{i=k}^{k+\alpha-1}\left(\left|\zeta_{i}\right|^{q}\left[r_{i}^{1-q}+h_{i}\right]-\left|\xi_{k}\right|^{q} r_{i}^{1-q}\right), \quad k \geq k_{0} \text {, }
\end{aligned}
$$

where the sequence of $h_{i}$ has the property that $\lim _{i \rightarrow \infty} h_{i}=0$. From (48), it follows

$$
\lim _{i \rightarrow \infty}\left|\zeta_{i}\right|^{q} h_{i}=0
$$

and, from (51), we obtain

$$
\left|\zeta_{i}-\xi_{k}\right|<\frac{(\alpha-1) K}{k-p+2}, \quad k \geq \widetilde{k}, i \in\{k, \ldots, k+\alpha-1\} .
$$

Since the function $y=|x|^{q}$ is continuously differentiable on $[-L, 0]$, there exists $N>0$ for which

$$
\left.|| \xi\right|^{q}-|\zeta|^{q}|\leq N| \xi-\zeta \mid, \quad \xi, \zeta \in[-L, 0] .
$$

Now it suffices to consider (32), (48), (53), (61), (62), (63), and (64) which imply the existence of an integer $\widehat{k} \geq \widetilde{k}$ such that

$$
\begin{aligned}
& \left|\frac{1}{\alpha} \sum_{i=k}^{k+\alpha-1} \frac{(i+1)(p-1)\left|\beta_{i}\right|^{p-2}\left|\zeta_{i}\right|^{q}}{[i(p-1)]^{q-1} \Phi\left[\Phi^{-1}\left(r_{i}\right)+\Phi^{-1}\left(-\zeta_{i} / i^{(p-1)}\right)\right]}-\frac{Y_{k}^{q}}{q}\right| \\
& \quad<\frac{M\left(\left\{s_{k}\right\}\right)[\gamma-\Gamma]}{8}, \quad k \geq \hat{k} .
\end{aligned}
$$

Altogether (see (54), (57), (59), and (65)), we have

$$
\begin{aligned}
\Delta \xi_{k} & >\frac{0+M\left(\left\{s_{k}\right\}\right)[\gamma-\Gamma] / 4-M\left(\left\{s_{k}\right\}\right)[\gamma-\Gamma] / 8}{k+\alpha-p+1} \\
& =\frac{M\left(\left\{s_{k}\right\}\right)[\gamma-\Gamma]}{8(k+\alpha-p+1)}, \quad k \geq \widehat{k} .
\end{aligned}
$$

Summing (66) from $\widehat{k}$ to an integer $l \geq \widehat{k}$, we obtain

$$
\xi_{l+1}>\xi_{\widehat{k}}+\frac{M\left(\left\{s_{k}\right\}\right)[\gamma-\Gamma]}{8} \sum_{k=\widehat{k}}^{l} \frac{1}{k+\alpha-p+1} .
$$

Thus (see also (53)), it is valid that

$$
\liminf _{l \rightarrow \infty} \xi_{l+1} \geq-L+\frac{M\left(\left\{s_{k}\right\}\right)[\gamma-\Gamma]}{8} \sum_{k=\widehat{k}}^{\infty} \frac{1}{k+\alpha-p+1}=\infty .
$$

Particularly (consider (52)), $\zeta_{k}$ has to be positive for infinitely many $k$. This contradiction proves that (34) is oscillatory if $\gamma>\Gamma$.

Let $\gamma<\Gamma$, and let $k(1) \in \mathbb{N}$ be given. In this part of the proof, our objective is to show that there exists a negative solution $\left\{\zeta_{k}\right\}$ of (39) for all $k \geq k(1)$, where

$$
\zeta_{k(1)}=-\left(\frac{q}{\alpha} \sum_{i=1}^{\alpha} r_{i}^{1-q}\right)^{1-p}
$$

Evidently, it is true that

$$
\Delta \zeta_{k} \geq \frac{1}{k-p+2}\left[(p-1) \zeta_{k}\right]
$$

$$
\begin{aligned}
& \Delta \zeta_{k} \\
& \leq \frac{1}{k-p+2} \\
& \quad \times\left[\gamma s^{+}+\frac{(k+1)(p-1)\left|\beta_{k}\right|^{p-2}\left|\zeta_{k}\right|^{q}}{\left[k^{(p-1)}\right]^{q-1} \Phi\left[\Phi^{-1}\left(r_{k}\right)+\Phi^{-1}\left(-\zeta_{k} / k^{(p-1)}\right)\right]}\right]
\end{aligned}
$$

for all $k \geq k(1)$ for which $\left\{\zeta_{k}\right\}$ is negative. Thus (consider (41), (42), and (44)), we can assume that $k(1)$ is so large that

$$
2 \zeta_{k(1)}<\zeta_{k(1)+i}<0, \quad i \in\{1, \ldots, \alpha\} .
$$

In addition, as in the first part of the proof (see (49)), we can estimate

$$
\left|\Delta \zeta_{k(1)+i}\right|<\frac{A}{k(1)-p+2}, \quad i \in\{0, \ldots, \alpha-2\},
$$

for some $A>0$. From (72), it follows

$$
\left|\zeta_{k(1)+i}-\zeta_{k(1)+j}\right|<\frac{A(\alpha-1)}{k(1)-p+2}, \quad i, j \in\{0, \ldots, \alpha-1\} .
$$

If we denote

$$
\xi_{k(1)}:=\frac{1}{\alpha} \sum_{i=k(1)}^{k(1)+\alpha-1} \zeta_{i}
$$

then

$$
\left|\zeta_{k(1)+i}-\xi_{k(1)}\right|<\frac{A(\alpha-1)}{k(1)-p+2}, \quad i \in\{0, \ldots, \alpha-1\} .
$$


Again (see (54)), we estimate

$$
\begin{aligned}
& \Delta \xi_{k(1)} \\
& \leq \frac{1}{k(1)-p+2} \\
& \quad \times\left[(p-1) \xi_{k(1)}+\frac{X_{k(1)}^{p}+\frac{Y_{k(1)}^{q}}{p}+\frac{\gamma^{k(1)+\alpha-1}}{\alpha} \sum_{i=k(1)} s_{i}-\frac{X_{k(1)}^{p}}{p}}{\quad+\frac{1}{\alpha} \sum_{i=k(1)}^{k(1)+\alpha-1} \frac{(i+1)(p-1)\left|\beta_{i}\right|^{p-2}\left|\zeta_{i}\right|^{q}}{[i(p-1)]^{q-1} \Phi\left[\Phi^{-1}\left(r_{i}\right)+\Phi^{-1}\left(-\zeta_{i} / i^{(p-1)}\right)\right]}}\right. \\
& \left.\quad-\frac{Y_{k(1)}^{q}}{q}\right]
\end{aligned}
$$

if $\Delta \xi_{k(1)}>0$, or

$$
\begin{aligned}
& \Delta \xi_{k(1)} \\
& \leq \frac{1}{k(1)+\alpha-p+1} \\
& \quad \times\left[(p-1) \xi_{k(1)}+\frac{X_{k(1)}^{p}}{p}\right. \\
& \quad+\frac{Y_{k(1)}^{q}+\frac{\gamma^{k(1)+\alpha-1}}{\alpha} \sum_{i=k(1)}^{q} s_{i}-\frac{X_{k(1)}^{p}}{p}}{\quad} \\
& \left.\left.\quad \frac{1}{\alpha} \sum_{i=k(1)}^{k(1)+\alpha-1} \frac{(i+1)(p-1)\left|\beta_{i}\right|^{p-2}\left|\zeta_{i}\right|^{q}}{[i(p-1)]^{q-1} \Phi\left[\Phi^{-1}\left(r_{i}\right)+\Phi^{-1}\left(-\zeta_{i} / i(p-1)\right.\right.}\right)\right] \\
& \left.\quad-\frac{Y_{k(1)}^{q}}{q}\right]
\end{aligned}
$$

if $\Delta \xi_{k(1)}<0$, where

$$
\begin{aligned}
X_{k(1)} & :=(p-1)\left(\frac{p^{k}}{\alpha} \sum_{i=k(1)}^{k(1)+\alpha-1} r_{i}^{1-q}\right)^{-1 / q}, \\
Y_{k(1)} & :=\left|\xi_{k(1)}\right|\left(\frac{p}{\alpha} \sum_{i=k(1)}^{k(1)+\alpha-1} r_{i}^{1-q}\right)^{1 / q} .
\end{aligned}
$$

If

$$
\begin{gathered}
\sum_{i=k(1)}^{k(1)+\alpha-1} r_{i}^{1-q}=\sum_{i=1}^{\alpha} r_{i}^{1-q}, \\
\zeta_{k(1)}=\zeta_{k(1)+j}, \quad j \in\{1, \ldots, \alpha-1\},
\end{gathered}
$$

then it is valid that

$$
\begin{aligned}
(p-1) \xi_{k(1)}+\frac{X_{k(1)}^{p}}{p}+\frac{Y_{k(1)}^{q}}{q} & (p-1) \xi_{k(1)}+\frac{(p-1)^{p}}{p}\left(\frac{p}{\alpha} \sum_{i=1}^{\alpha} r_{i}^{1-q}\right)^{-p / q} \\
= & +\frac{\left|\xi_{k(1)}\right|^{q}}{q} \frac{p}{\alpha} \sum_{i=1}^{\alpha} r_{i}^{1-q}=-(p-1)\left(\frac{q}{\alpha} \sum_{i=1}^{\alpha} r_{i}^{1-q}\right)^{1-p} \\
& +\left(\frac{p-1}{p}\right)^{p}\left(\frac{1}{\alpha} \sum_{i=1}^{\alpha} r_{i}^{1-q}\right)^{-p / q}+\frac{\left((q / \alpha) \sum_{i=1}^{\alpha} r_{i}^{1-q}\right)^{q[1-p]}}{q} \\
& \times \frac{p}{\alpha} \sum_{i=1}^{\alpha} r_{i}^{1-q}=-(p-1) q^{1-p}\left(\frac{1}{\alpha} \sum_{i=1}^{\alpha} r_{i}^{1-q}\right)^{1-p} \\
& +\left(\frac{p-1}{p}\right)^{p}\left(\frac{1}{\alpha} \sum_{i=1}^{\alpha} r_{i}^{1-q}\right)^{1-p}+q^{-p-1} p\left(\frac{1}{\alpha} \sum_{i=1}^{\alpha} r_{i}^{1-q}\right)^{1-p}
\end{aligned}
$$

That is,

$$
\begin{aligned}
(p & -1) \xi_{k(1)}+\frac{X_{k(1)}^{p}}{p}+\frac{Y_{k(1)}^{q}}{q} \\
& =\left(\frac{1}{\alpha} \sum_{i=1}^{\alpha} r_{i}^{1-q}\right)^{1-p}\left[-(p-1) q^{1-p}+\left(\frac{p-1}{p}\right)^{p}+q^{-p-1} p\right] \\
& =\left(\frac{1}{\alpha} \sum_{i=1}^{\alpha} r_{i}^{1-q}\right)^{1-p}\left[-p q^{1-p}+q^{1-p}+\frac{1}{q^{p}}+q^{-p-1} p\right] \\
& =\left(\frac{1}{\alpha} \sum_{i=1}^{\alpha} r_{i}^{1-q}\right)^{1-p} q^{-p}\left[-p q+q+1+\frac{p}{q}\right] \\
& =\left(\frac{1}{\alpha} \sum_{i=1}^{\alpha} r_{i}^{1-q}\right)^{1-p} q^{-p}[-p q+q+1+p-1]=0 .
\end{aligned}
$$

For any $\varepsilon>0$, we can find $\alpha \in \mathbb{N}$ with the property that

$$
\left|\sum_{i=k}^{k+\alpha-1} r_{i}^{1-q}-\sum_{i=1}^{\alpha} r_{i}^{1-q}\right|<\varepsilon, \quad k \in \mathbb{N}
$$

Thus, for a given number $\alpha$, considering (73) and (79), we can assume that $k(1)$ is so large that

$$
\left|(p-1) \xi_{k(1)}+\frac{X_{k(1)}^{p}}{p}+\frac{Y_{k(1)}^{q}}{q}\right|<\frac{M\left(\left\{s_{k}\right\}\right)[\Gamma-\gamma]}{16} .
$$


It holds (see (36), (37), and (38))

$$
\begin{aligned}
& \frac{\gamma^{k}}{\alpha} \sum_{i=k(1)}^{k(1)+\alpha-1} s_{i}-\frac{X_{k(1)}^{p}}{p} \\
& =\frac{\gamma}{\alpha} \sum_{i=k(1)}^{k(1)+\alpha-1} s_{i}-\frac{(p-1)^{p}}{p}\left(\frac{p}{\alpha} \sum_{i=k(1)}^{k(1)+\alpha-1} r_{i}^{1-q}\right)^{-p / q} \\
& =\frac{1}{\alpha} \sum_{i=k(1)}^{k(1)+\alpha-1} s_{i}\left[\gamma-q^{-p}\left(\frac{1}{\alpha} \sum_{i=k(1)}^{k(1)+\alpha-1} r_{i}^{1-q}\right)^{1-p}\right. \\
& <\frac{M\left(\left\{s_{k}\right\}\right)}{2} \frac{\gamma-\Gamma}{2} .
\end{aligned}
$$

That is,

$$
\frac{\gamma^{k}}{\alpha} \sum_{i=k(1)}^{k(1)+\alpha-1} s_{i}-\frac{X_{k(1)}^{p}}{p}<\frac{M\left(\left\{s_{k}\right\}\right)[\gamma-\Gamma]}{4}<0 .
$$

As in the first part of the proof (compare (48) with (71) and (63) with (75)), we can show that

$$
\begin{aligned}
\lim _{k(1) \rightarrow \infty} \mid \frac{1}{\alpha} \sum_{i=k(1)}^{k(1)+\alpha-1} \frac{(i+1)(p-1)\left|\beta_{i}\right|^{p-2}\left|\zeta_{i}\right|^{q}}{\left[i^{(p-1)}\right]^{q-1} \Phi\left[\Phi^{-1}\left(r_{i}\right)+\Phi^{-1}\left(-\zeta_{i} / i^{(p-1)}\right)\right]} \\
-\frac{Y_{k(1)}^{q}}{q} \mid=0 .
\end{aligned}
$$

Let $k(1)$ be so large that

$$
\begin{aligned}
\mid \frac{1}{\alpha} \sum_{i=k(1)}^{k(1)+\alpha-1} \frac{(i+1)(p-1)\left|\beta_{i}\right|^{p-2}\left|\zeta_{i}\right|^{q}}{\left[i^{(p-1)}\right]^{q-1} \Phi\left[\Phi^{-1}\left(r_{i}\right)+\Phi^{-1}\left(-\zeta_{i} / i^{(p-1)}\right)\right]} \\
-\frac{Y_{k(1)}^{q}}{q} \mid<\frac{M\left(\left\{s_{k}\right\}\right)[\Gamma-\gamma]}{16} .
\end{aligned}
$$

Finally (consider (77), (83), (85), and (87)), we have

$$
\begin{aligned}
\Delta \xi_{k(1)}< & \frac{1}{k(1)+\alpha-p+1} \\
& \times\left(\frac{M\left(\left\{s_{k}\right\}\right)[\Gamma-\gamma]}{16}+\frac{M\left(\left\{s_{k}\right\}\right)[\gamma-\Gamma]}{4}\right. \\
& \left.+\frac{M\left(\left\{s_{k}\right\}\right)[\Gamma-\gamma]}{16}\right) \\
= & \frac{M\left(\left\{s_{k}\right\}\right)[\gamma-\Gamma]}{8(k(1)+\alpha-p+1)}<0 .
\end{aligned}
$$

Hence,

$$
\Delta \xi_{k(1)}=\frac{1}{\alpha} \sum_{i=k(1)}^{k(1)+\alpha-1} \Delta \zeta_{i}=\frac{\zeta_{k(1)+\alpha}-\zeta_{k(1)}}{\alpha}<0 .
$$

That is,

$$
\zeta_{k(1)+\alpha}<\zeta_{k(1)}
$$

Particularly, if

$$
\zeta_{k}=-\left(\frac{q}{\alpha} \sum_{i=1}^{\alpha} r_{i}^{1-q}\right)^{1-p} \quad \text { for some } k \geq k(1),
$$

then (71) and (90) give

$$
\zeta_{k}, \zeta_{k+1}, \ldots, \zeta_{k+\alpha-1}<0, \quad \zeta_{k+\alpha}<\zeta_{k}
$$

Since (73) remains true if $k(1)$ is replaced by an arbitrary integer $k>k(1)$, to complete the proof, it suffices to find a number $\vartheta>0$ such that

$$
\begin{aligned}
\zeta_{k} \in(- & 9-\left(\frac{q}{\alpha} \sum_{i=1}^{\alpha} r_{i}^{1-q}\right)^{1-p}, \\
& \left.-\left(\frac{q}{\alpha} \sum_{i=1}^{\alpha} r_{i}^{1-q}\right)^{1-p}\right) \text { for some } k \geq k(1)
\end{aligned}
$$

implies

$$
\frac{1}{\alpha} \sum_{i=k}^{k+\alpha-1} \Delta \zeta_{i}<0
$$

Indeed, in this case, we have (92) and, consequently, we have that $\zeta_{k}<0$ for all $k>k(1)$. Then, Lemma 16 says that (34) is nonoscillatory. Of course, such a number $\vartheta>0$ exists because the concrete initial value is not applied to obtain (77), (85), and (87) and because (83) can be proved for a sufficiently small perturbation of the initial value (the left side of (83) continuously depends on the initial value (69))

Remark 18. The statement of Theorem 17 remains true for $\gamma \in$ $\mathbb{R}$. It suffices to consider the nonoscillatory equation

$$
\Delta\left[r^{-} \Phi\left(\Delta x_{k}\right)\right]+0 \cdot \Phi\left(x_{k+1}\right)=0,
$$

where $r^{-}$is defined in (35). We refer to [1, Theorem 2].

Remark 19. We conjecture that, for $\gamma=\Gamma$ in the general case, it is not possible to decide whether (34) is oscillatory or nonoscillatory. Our conjecture is based on the existence of positive almost periodic sequences $\left\{f_{k}\right\}_{k \in \mathbb{Z}},\left\{g_{k}\right\}_{k \in \mathbb{Z}}$ satisfying

$$
\begin{gathered}
\frac{1}{n} \sum_{k=1}^{n} f_{k}<M\left(\left\{f_{k}\right\}\right), \\
\frac{1}{n} \sum_{k=1}^{n} g_{k}>M\left(\left\{g_{k}\right\}\right), \quad n \in \mathbb{N} .
\end{gathered}
$$

Such sequences can be constructed applying, for example, [34, Theorem 3.5]. 
Example 20. Let $a>0, b>1 / 2$, and $c, d \neq 0$. We can use Theorem 17 for the following equation:

$$
\begin{aligned}
& \Delta\left[\frac{k^{2}}{1+k^{2}(\cos [a k] \sin [a k]+b)} \Phi\left(\Delta x_{k}\right)\right] \\
& \quad+\gamma \frac{\sqrt[k]{k}-1+|\sin [c k]|+|\cos [d k]|}{(k+1)^{(3 / 2)}} \Phi\left(x_{k+1}\right)=0
\end{aligned}
$$

with asymptotically almost periodic coefficients (consider Definition 2, Theorem 3, and Corollaries 6 and 13). It holds $(p=3 / 2$, i.e., $q=3)$

$$
\begin{aligned}
& M\left(\left\{r_{k}^{-2}\right\}\right)=M\left(\left\{\left(\cos [a k] \sin [a k]+b+\frac{1}{k^{2}}\right)^{2}\right\}\right) \\
&=\frac{1}{8}+b^{2}, \\
& M\left(\left\{s_{k}\right\}\right)=M(\{\sqrt[k]{k}-1+|\sin [c k]|+|\cos [d k]|\})=\frac{4}{\pi}
\end{aligned}
$$

Thus, the equation is oscillatory for

$$
6 \gamma \sqrt{3\left(8 b^{2}+1\right)}>\sqrt{2} \pi
$$

and nonoscillatory for

$$
6 \gamma \sqrt{3\left(8 b^{2}+1\right)}<\sqrt{2} \pi .
$$

Theorem 17 gives a new result also for $p=2$ (i.e., for the linear equations with asymptotically almost periodic coefficients). This situation is illustrated by the next example. Note that, in the below given corollaries, we explicitly mention two other new results which follow from Theorem 17.

Example 21. For any positive continuous functions $f:[-2$, $2] \rightarrow \mathbb{R}$ and $g:[-1,1] \rightarrow \mathbb{R}$, the equation

$$
\Delta\left[f\left(\sin (1-k)+\frac{(-1)^{k}}{\sqrt{k}}\right) \Delta x_{k}\right]+\gamma \frac{k g(\cos k)+1}{(k+1) k^{2}} x_{k+1}=0
$$

has the form of (34) for $p=q=2$. Considering [29, Example 3.4], we have

$$
\begin{aligned}
& M\left(\left\{r_{k}^{-1}\right\}\right)=\frac{1}{2 \pi} \int_{-\pi}^{\pi} \frac{d t}{f(\sin t)}, \\
& M\left(\left\{s_{k}\right\}\right)=\frac{1}{2 \pi} \int_{-\pi}^{\pi} g(\cos t) d t .
\end{aligned}
$$

Hence, the oscillation constant is

$$
\Gamma=\left[\frac{1}{\pi^{2}} \int_{-\pi}^{\pi} \frac{d t}{f(\sin t)} \int_{-\pi}^{\pi} g(\cos t) d t\right]^{-1} .
$$

Corollary 22. Consider (34), where $\left\{r_{k}\right\}_{k \in \mathbb{N}}$ and $\left\{s_{k}\right\}_{k \in \mathbb{N}}$ are positive periodic sequences with period $n$. Denote

$$
\Gamma:=q^{-p}\left[\frac{1}{n} \sum_{i=1}^{n} r_{i}^{1-q}\right]^{1-p}\left[\frac{1}{n} \sum_{i=1}^{n} s_{i}\right]^{-1} .
$$

Then, (34) is oscillatory for $\gamma>\Gamma$ and nonoscillatory for $\gamma<\Gamma$.
Example 23. We apply Corollary 22 for the equation

$$
\begin{aligned}
& \Delta\left[\left|\sin \frac{(2 k-1) \pi}{18}\right| \Phi\left(\Delta x_{k}\right)\right] \\
& +\frac{\gamma}{(k+1)^{(p)}}\left(1+\cos \frac{2 k \pi}{m}\right) \Phi\left(x_{k+1}\right)=0,
\end{aligned}
$$

where $m \geq 3$ is an odd integer. By the following computations:

$$
\begin{aligned}
& M\left(\left\{r_{k}^{1-q}\right\}\right)=\frac{1}{9} \sum_{i=1}^{9} r_{i}^{1-q}=\frac{1}{9} \sum_{i=1}^{9}\left|\sin \frac{(2 i-1) \pi}{18}\right|^{1-q} \\
& =\frac{1}{9}\left(2^{q}+2 \sin ^{1-q} \frac{\pi}{18}+2 \cos ^{1-q} \frac{\pi}{9}\right. \\
& \left.+2 \cos ^{1-q} \frac{2 \pi}{9}+1\right) \\
& M\left(\left\{s_{k}\right\}\right)=\frac{1}{m} \sum_{i=1}^{m} s_{i}=\frac{1}{m} \sum_{i=1}^{m}\left(1+\cos \frac{2 i \pi}{m}\right)=1
\end{aligned}
$$

we get the oscillation constant

$$
\begin{aligned}
\Gamma=9^{p-1} q^{-p}\left(2^{q}\right. & +2 \sin ^{1-q} \frac{\pi}{18} \\
& \left.+2 \cos ^{1-q} \frac{\pi}{9}+2 \cos ^{1-q} \frac{2 \pi}{9}+1\right)^{1-p} .
\end{aligned}
$$

For the general nonzero sequence $\left\{r_{k}\right\}_{k \in \mathbb{N}}$ in (34), we have to use the full definition of the generalized zero. An interval $(l, l+1], l \in \mathbb{N}$, contains the generalized zero of a solution $\left\{x_{k}\right\}$ of (34) if $x_{l} \neq 0$ and $r_{l} x_{l} x_{l+1} \leq 0$. This definition is necessary for the correct formulation of Corollary 24 .

Corollary 24. Consider (34), where $\left\{r_{k}\right\}_{k \in \mathbb{N}}$ and $\left\{s_{k}\right\}_{k \in \mathbb{N}}$ are nonzero asymptotically almost periodic sequences satisfying

$$
\inf \left\{\left|r_{k}\right| ; k \in \mathbb{N}\right\}>0, \quad \limsup _{k \rightarrow \infty}\left|s_{k}\right|>0 \text {. }
$$

Let

$$
\Gamma:=q^{-p}\left[M\left(\left\{\left|r_{k}\right|^{1-q}\right\}\right)\right]^{1-p}\left[M\left(\left\{\left|s_{k}\right|\right\}\right)\right]^{-1}
$$

(i) If $r_{k}>0$ for all $k$ and $\gamma<\Gamma$, then (34) is nonoscillatory.

(ii) If $s_{k}>0$ for all $k$ and $\gamma>\Gamma$, then (34) is oscillatory.

Proof. Since $\left\{\left|f_{k}\right|\right\}_{k \in \mathbb{N}}$ is asymptotically almost periodic if $\left\{f_{k}\right\}_{k \in \mathbb{N}}$ is asymptotically almost periodic, it suffices to use the half-linear Sturm type comparison theorem (see [1, Theorem 2]) and Theorem 17.

Example 25. For an arbitrarily given $a \geq 1$, Corollary 24 says that the equation

$$
\Delta\left[\left(\frac{\cos k}{k}+a\right) \Phi\left(\Delta x_{k}\right)\right]+\frac{\gamma \sin k}{(k+1)^{(p)}} \Phi\left(x_{k+1}\right)=0
$$


is nonoscillatory if

$$
\gamma<q^{-p}\left[a^{1-q}\right]^{1-p}[M(\{|\sin k|\})]^{-1}=q^{-p} \frac{a \pi}{2}
$$

and that the equation

$$
\Delta\left[(-1)^{k}\left(\frac{\cos k}{k}+a\right) \Phi\left(\Delta x_{k}\right)\right]+\frac{\gamma|\sin k|}{(k+1)^{(p)}} \Phi\left(x_{k+1}\right)=0
$$

is oscillatory if

$$
\gamma>q^{-p}\left[a^{1-q}\right]^{1-p}[M(\{|\sin k|\})]^{-1}=q^{-p} \frac{a \pi}{2} .
$$

\section{Acknowledgments}

The authors thank the referees for their remarks and suggestions which improved the final version of the paper. The first author is supported by the Programme of "Employment of Best Young Scientists for International Cooperation Empowerment" (grant number CZ.1.07/2.3.00/30.0037) cofinanced from European Social Fund and the state budget of the Czech Republic. The second author is supported by the Czech Science Foundation under Grant P201/10/1032.

\section{References}

[1] P. Řehák, "Oscillatory properties of second order half-linear difference equations," Czechoslovak Mathematical Journal, vol. 51, no. 2, pp. 303-321, 2001.

[2] O. Došlý and S. Fišnarová, "Linearized Riccati technique and (non-)oscillation criteria for half-linear difference equations," Advances in Difference Equations, vol. 2008, Article ID 438130, 18 pages, 2008.

[3] O. Došlý and P. Řehák, "Nonoscillation criteria for half-linear second-order difference equations," Computers \& Mathematics with Applications, vol. 42, no. 3-5, pp. 453-464, 2001.

[4] H. A. El-Morshedy, "Oscillation and nonoscillation criteria for half-linear second order difference equations," Dynamic Systems and Applications, vol. 15, no. 3-4, pp. 429-450, 2006.

[5] J. Jiang and X. Tang, "Oscillation of second order half-linear difference equations (II)," Applied Mathematics Letters of Rapid Publication, vol. 24, no. 9, pp. 1495-1501, 2011.

[6] P. Řehák, "Generalized discrete Riccati equation and oscillation of half-linear difference equations," Mathematical and Computer Modelling, vol. 34, no. 3-4, pp. 257-269, 2001.

[7] P. Řehák, "Oscillation criteria for second order half-linear difference equations," Journal of Difference Equations and Applications, vol. 7, no. 4, pp. 483-505, 2001.

[8] Y. G. Sun and F. W. Meng, "Nonoscillation and oscillation of second order half-linear difference equations," Applied Mathematics and Computation, vol. 197, no. 1, pp. 121-127, 2008.

[9] E. Thandapani, K. Ravi, and J. R. Graef, "Oscillation and comparison theorems for half-linear second-order difference equations," Computers \& Mathematics with Applications, vol. 42, no. 6-7, pp. 953-960, 2001.

[10] O. Došlý and S. Fišnarová, "Two-parametric conditionally oscillatory half-linear differential equations," Abstract and Applied Analysis, vol. 2011, Article ID 182827, 16 pages, 2011.
[11] O. Došlý and H. Funková, "Perturbations of half-linear Euler differential equation and transformations of modified Riccati equation," Abstract and Applied Analysis, vol. 2012, Article ID 738472, 19 pages, 2012.

[12] S. Fišnarová and R. Mařík, "On constants in nonoscillation criteria for half-linear differential equations," Abstract and Applied Analysis, vol. 2011, Article ID 638271, 15 pages, 2011.

[13] O. Došlý, J. R. Graef, and J. Jaroš, "Forced oscillation of second order linear and half-linear difference equations," Proceedings of the American Mathematical Society, vol. 131, no. 9, pp. 28592867, 2003.

[14] P. J. Y. Wong and R. P. Agarwal, "Oscillations and nonoscillations of half-linear difference equations generated by deviating arguments," Computers \& Mathematics with Applications, vol. 36, no. 10-12, pp. 11-26, 1998.

[15] R. P. Agarwal, M. Bohner, S. R. Grace, and D. O’Regan, Discrete Oscillation Theory, Hindawi Publishing Corporation, New York, NY, USA, 2005.

[16] P. R. Beesack, "Hardy's inequality and its extensions," Pacific Journal of Mathematics, vol. 11, pp. 39-61, 1961.

[17] Á. Elbert, "A half-linear second order differential equation," in Qualitative Theory of Differential Equations, Vol. I, II (Szeged, 1979), vol. 30 of Colloquia Mathematica Societatis János Bolyai, pp. 153-180, North-Holland, Amsterdam, The Netherlands, 1981.

[18] J. D. Mirzov, “On some analogs of Sturm's and Kneser's theorems for nonlinear systems," Journal of Mathematical Analysis and Applications, vol. 53, no. 2, pp. 418-425, 1976.

[19] O. Došlý and P. Řehák, Half-Linear Differential Equations, Elsevier, Amsterdam, The Netherlands, 2005.

[20] P. Hasil and M. Veselý, "Oscillation of half-linear differential equations with asymptotically almost periodic coefficients," Advances in Difference Equations, vol. 2013, no. 122, pp. 1-15, 2013.

[21] R. P. Agarwal, Difference Equations and Inequalities: Theory, Methods, and Applications, Marcel Dekker, New York, NY, USA, 2000.

[22] W. G. Kelley and A. C. Peterson, Difference Equations: An Introduction with Applications, Academic Press, San Diego, Calif, USA, 2001.

[23] A. Kneser, "Untersuchungen über die reellen Nullstellen der Integrale linearer Differentialgleichungen," Mathematische Annalen, vol. 42, no. 3, pp. 409-435, 1893.

[24] P. B. Naĭman, "The set of isolated points of increase of the spectral function pertaining to a limit-constant Jacobi matrix," Izvestija Vysših Učebnyh Zavedeniĭ Matematika, vol. 1959, no. 1 (8), pp. 129-135, 1959 (Russian).

[25] F. Gesztesy and M. Ünal, "Perturbative oscillation criteria and Hardy-type inequalities," Mathematische Nachrichten, vol. 189, pp. 121-144, 1998.

[26] K. M. Schmidt, "Critical coupling constants and eigenvalue asymptotics of perturbed periodic Sturm-Liouville operators," Communications in Mathematical Physics, vol. 211, no. 2, pp. 465-485, 2000.

[27] H. Krüger, "On perturbations of quasiperiodic Schrödinger operators," Journal of Differential Equations, vol. 249, no. 6, pp. 1305-1321, 2010.

[28] H. Krüger and G. Teschl, "Effective Prüfer angles and relative oscillation criteria," Journal of Differential Equations, vol. 245, no. 12, pp. 3823-3848, 2008. 
[29] P. Hasil and M. Veselý, "Critical oscillation constant for difference equations with almost periodic coefficients," Abstract and Applied Analysis, vol. 2012, Article ID 471435, 19 pages, 2012.

[30] F. Luef and G. Teschl, "On the finiteness of the number of eigenvalues of Jacobi operators below the essential spectrum," Journal of Difference Equations and Applications, vol. 10, no. 3, pp. 299-307, 2004.

[31] P. Hasil, "Conditional oscillation of half-linear differential equations with periodic coefficients," Archivum Mathematicum, vol. 44, no. 2, pp. 119-131, 2008.

[32] O. Došlý and P. Hasil, "Critical oscillation constant for halflinear differential equations with periodic coefficients," Annali di Matematica Pura ed Applicata IV, vol. 190, no. 3, pp. 395-408, 2011.

[33] C. Corduneanu, Almost Periodic Functions, John Wiley \& Sons, New York, NY, USA, 1968.

[34] M. Veselý, "Construction of almost periodic sequences with given properties," Electronic Journal of Differential Equations, vol. 2008, no. 126, pp. 1-22, 2008.

[35] K. Fan, "Les fonctions asymptotiquement presque-périodiques d'une variable entière et leur application à l'étude de l'itération des transformations continues," Mathematische Zeitschrift, vol. 48, pp. 685-711, 1943.

[36] P. Řehák, "Hartman-Wintner type lemma, oscillation, and conjugacy criteria for half-linear difference equations," Journal of Mathematical Analysis and Applications, vol. 252, no. 2, pp. 813-827, 2000. 


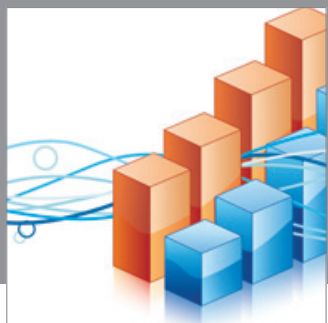

Advances in

Operations Research

mansans

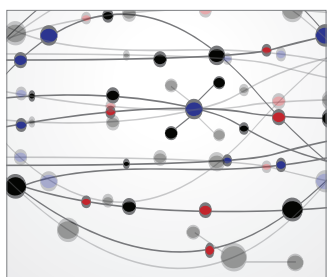

The Scientific World Journal
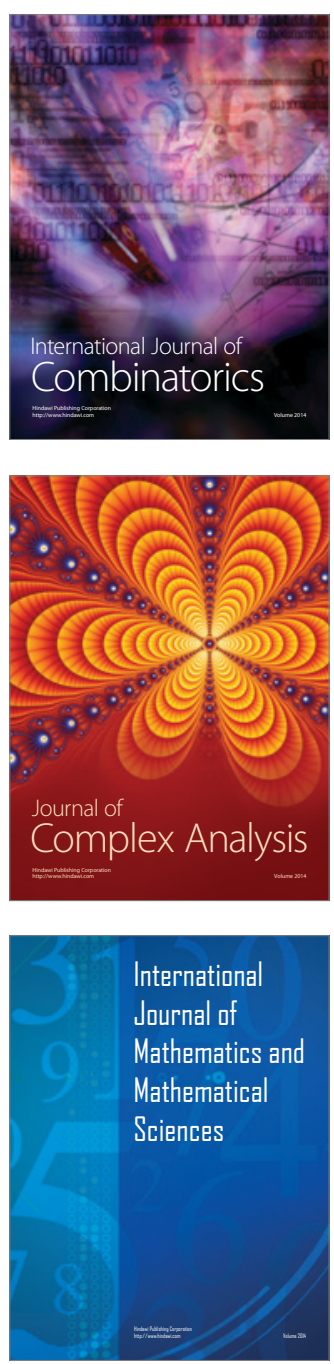
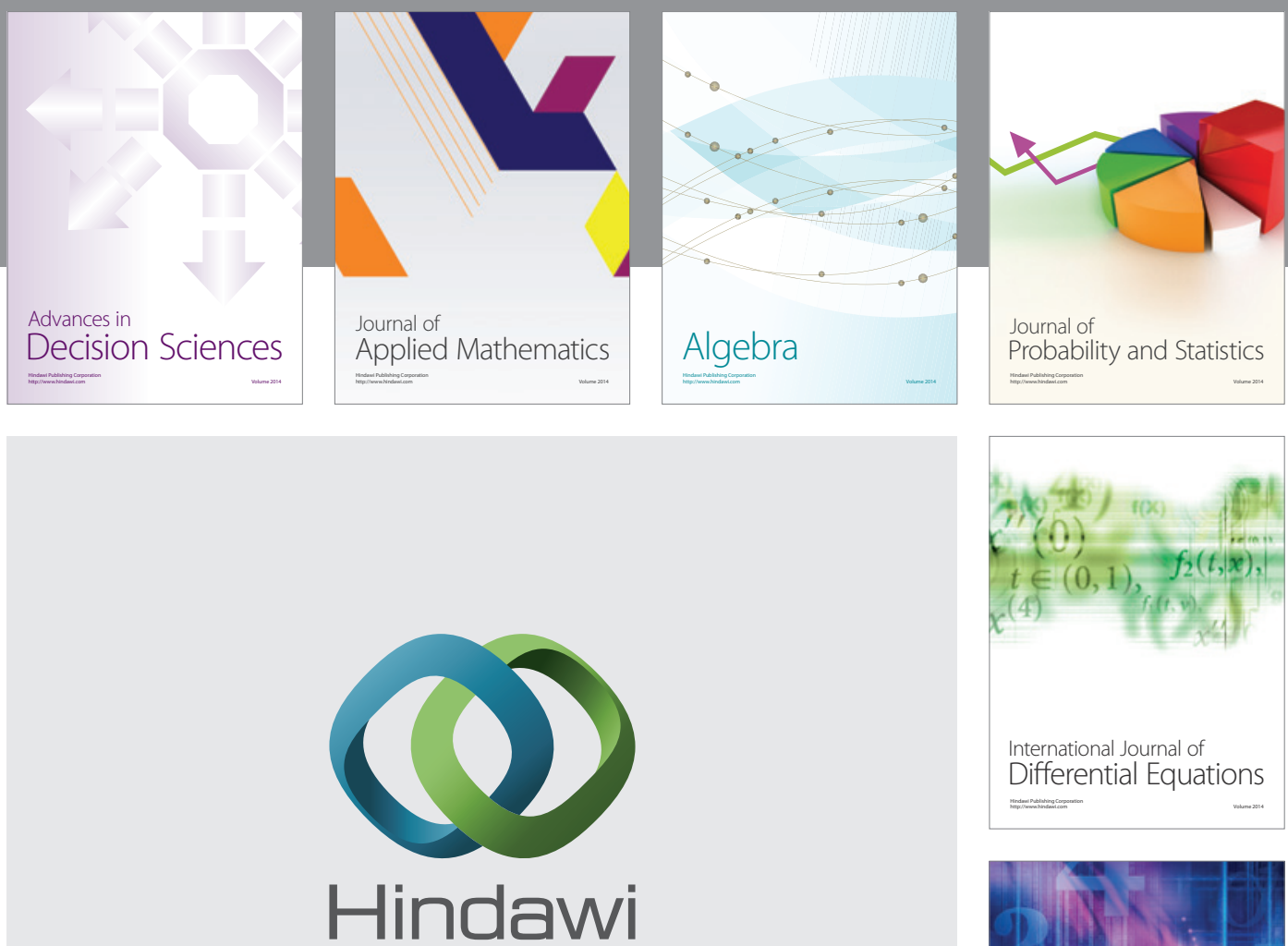

Submit your manuscripts at http://www.hindawi.com
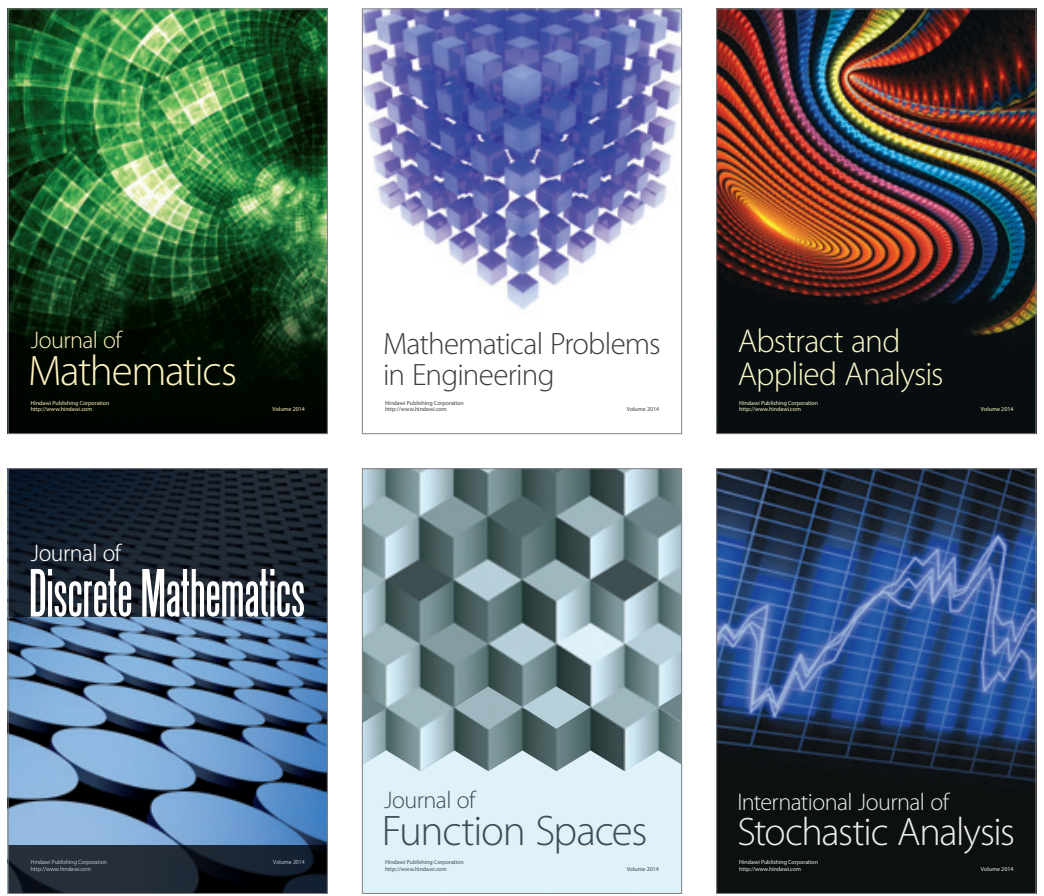

Journal of

Function Spaces

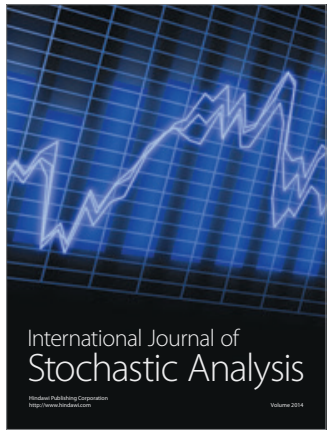

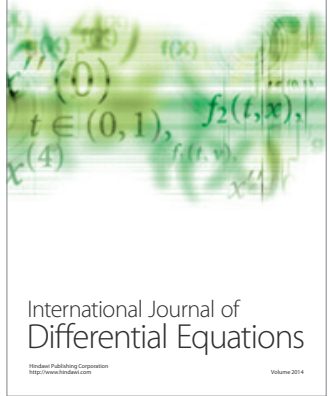
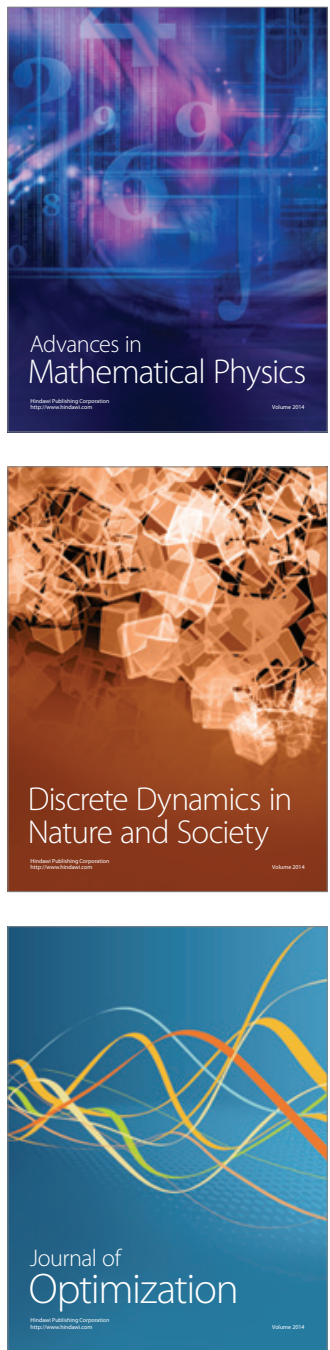